\title{
Minimizing Charging Delay in Wireless Rechargeable Sensor Networks
}

\author{
Lingkun $\mathrm{Fu}^{* \dagger}$, Peng Cheng* ${ }^{* \dagger}$, Yu Gu${ }^{\dagger}$, Jiming Chen*, Tian $\mathrm{He}^{\S}$ \\ ${ }^{*}$ State Key Laboratory of Industrial Control Technology, Zhejiang University, China \\ ${ }^{\dagger}$ Singapore University of Technology and Design, Singapore \\ ${ }^{\S}$ Dept. of Computer Science and Engineering, University of Minnesota, USA \\ Email: \{lkfu,pcheng,jmchen\}@iipc.zju.edu.cn, jasongu@ sutd.edu.sg, tianhe@cs.umn.edu
}

\begin{abstract}
As a pioneering experimental platform of wireless rechargeable sensor networks, the Wireless Identification and Sensing Platform (WISP) is an open-source platform that integrates sensing and computation capabilities to the traditional RFID tags. Different from traditional tags, a RFID-based wireless rechargeable sensor node needs to charge its onboard energy storage above a threshold in order to power its sensing, computation and communication components. Consequently, such charging delay imposes a unique design challenge for deploying wireless rechargeable sensor networks. In this paper, we tackle this problem by planning the optimal movement strategy of the RFID reader, such that the time to charge all nodes in the network above their energy threshold is minimized. We first propose an optimal solution using the linear programming method. To further reduce the computational complexity, we then introduce a heuristic solution with a provable approximation ratio of $(1+\theta) /(1-\varepsilon)$ by discretizing the charging power on a twodimensional space. Through extensive evaluations, we demonstrate that our design outperforms the set-cover-based design by an average of $24.7 \%$ while the computational complexity is $\mathcal{O}\left((N / \varepsilon)^{2}\right)$.
\end{abstract}

\section{INTRODUCTION}

Energy is by far one of the most critical design hurdles that hindering the deployment of wireless sensor networks [1][4]. As most existing sensor network systems are powered by batteries, their lifetime are limited by the storage capacity of the battery used. The recent breakthrough in wireless energy transfer technology developed by Kurs et al. [5] provides a promising alternative to power these sensor nodes. We expect in the near future a new class of Wireless Rechargeable Sensor Networks will have potentials to bring universal sensing, communication and computation capabilities to our daily life. For example, without batteries attached to a node, we can design much smaller and more flexible sensor nodes that can be attached to objects such as fruit and medical pills, which are not traditionally instrumented. Clearly, by providing realtime monitoring capabilities to our critical daily products, we have the potential to significantly improve the quality of living for the general public.

Recently, to improve the energy charging efficiency for wireless rechargeable sensor networks, many works have focused on the internal microelectronics design [6]-[8]. While it is essential to improve the fundamental microelectronics design for wireless energy transfer systems, we observe the practical applications of wireless rechargeable sensor networks that the charging time of individual wireless rechargeable sensor nodes is also not negligible and plays an important role in the overall system performance. For a typical wireless rechargeable sensor node, such as Intel Research's Wireless Identification and Sensing Platform (WISP) [9], the sensor node has to be wireless charged above a certain threshold in order for various sensing, computation and communication components to function properly. For example, through our empirical measurement, we observe that the charging time for voltage to reach $1.8 \mathrm{~V}$ to power a WISP equipped with a $100 u F$ capacitor can be as large as 155 seconds, when the RFID reader is 10.0 meters away.

As RFID readers are normally much more expensive than wireless rechargeable sensor nodes (about 100 times price difference [10]), for a practical wireless rechargeable sensor networks, we would normally need one or more readers that are carried by robots [11]-[13] or move along an existing infrastructure such as moving tracks to efficiently power all nodes in the network [10]. Therefore, it is essential to plan the movement pattern of RFID readers so as to minimize the total charging delay of the network.

In this paper, we consider the scenario that a RFID reader moves and stops at several locations to wireless charge nodes and obtain readings of interests at its surroundings. Such scenario can be applied to various types of industry settings, for example, in warehouse inventory management [14] or in large distribution center [10]. Our optimization object, therefore, is to identify the optimal reader stop locations and the corresponding stop durations such that the total delay to charge all nodes in the network above their energy threshold is minimized. The major contributions of this paper are as follows:

- We identify charging delay as one of the key design hurdles in wireless rechargeable sensor networks and introduce an effective solution to minimize charging delay in such networks. To the best of our knowledge, this is the first work that provides a general mean to minimize charging delay in wireless rechargeable sensor networks.

- We formulate the charging delay minimization problem as a linear programming problem, which can be optimally solved to identify the optimal reader stop locations and the corresponding stop durations.

- We introduce the concepts of the smallest enclosing 


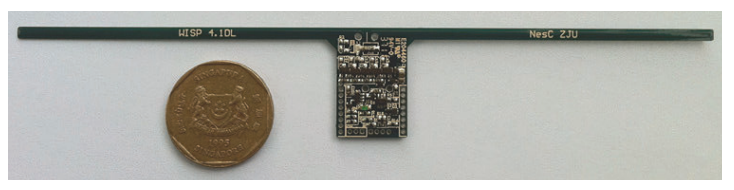

Fig. 1. WISP node fabricated in our lab

space and charging power discretization to reduce the computational complexity, which significantly reduce the searching space for the optimal solution with a $1 /(1-\varepsilon)$ approximation ratio.

- The optimal solution obtained by above designs may consist a large number of stop locations and incur a long travel distance of RFID reader. For practical consideration, we further introduce a location merging scheme to reduce the number of stop locations while maintaining the charging delay to a $(1+\theta)$ upper bound.

The rest of this paper is organized as follows. Section II describes our energy rechargeable platform and its charging model. In Section III we introduce our problem formulation and present a heuristic solution to the problem. We evaluate our design in Section IV. Section V discusses related works and we conclude the paper in Section VI.

\section{PRELIMINARIES}

\section{A. Wireless Identification and Sensing Platform (WISP)}

Wireless Identification and Sensing Platform (WISP), developed by Intel Research [9], is one of the most representative wireless rechargeable sensor node platform. WISP node inherits the capabilities of traditional RFID tags, but also supports sensing and computing. When near to a RFID reader, WISP node can harvest energy from the reader signals. The charged energy is stored in a capacitor and can be used for future data sensing, logging, computing, and transferring [15]. Fig. 1 shows a customized WISP node with different system components and energy storage devices in our lab. The size of WISP node is similar to a SGD \$1 dollar coin, and it can be easily attached to containers, packages or other daily objects.

\section{B. Energy Charging Model}

In this work, we use the WISP-reader charging model proposed in [16] as follows:

$$
P_{r}=\frac{G_{s} G_{r} \eta}{L_{p}}\left(\frac{\lambda}{4 \pi(d+\beta)}\right)^{2} P_{0},
$$

where $d$ is the distance between the sensor node and RFID reader, $P_{0}$ is the source power, $G_{s}$ is the source antenna gain, $G_{r}$ is the receive antenna gain, $L_{p}$ is polarization loss, $\lambda$ is the wavelength, $\eta$ is rectifier efficiency, and $\beta$ is a parameter to adjust the Friis' free space equation for short distance transmission. Except for distance $d$, all other parameters in Eq. 1 are constant values based on the environment and device settings. This model is based on the Friis' free space equation and has been experimentally shown to be a good approximation of charged energy in [16]. To further validate this charging model, we perform additional experiments to investigate the charged power by varying distances between

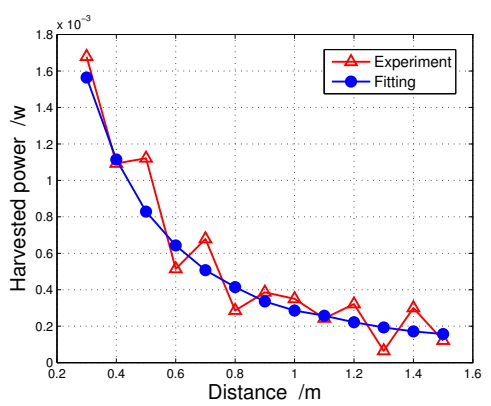

Fig. 2. Experiment and energy charging model for the harvested power under different distances between reader and sensor node with source power is $1 \mathrm{~W}$

the reader and sensor node. As shown in Fig. 2, the measured harvested power matches Eq. 1 well during our experiments.

To ease the description of design in the following sections, we simplify the charging model in Eq. 1 as:

$$
P_{r}=\frac{\alpha}{(d+\beta)^{2}},
$$

where $d$ is the distance from a sensor node to the reader, and $\alpha$ represents other constant environment parameters including $P_{0}, G_{s}, G_{r}, L_{p}, \lambda$, and $\eta$ in Eq. 1.

Here we would emphasize that our design does not depend on any specific charging model. As long as the charging power shows a trend of negative correlation with the charging distance, which is true in most practical settings [5], [17], our work can be directly applied under these models.

\section{MAIN DESIGN}

\section{A. Problem Formulation}

We assume a network deployment with $N$ stationary wireless rechargeable sensor nodes. The location of a node $i$ in the network can be localized using techniques in [18]-[20] and represented as $\left(W_{x}^{i}, W_{y}^{i}\right)$. We also assume there is a RFID reader that is able to move around with a robot [11]-[13] or along an existing infrastructure (moving tracks and etc.) [10]. When nodes are near to a RFID reader, they charge their internal energy storages and are able to perform functionalities such as sensing, computation and communication when the charged energy is above a threshold $\delta$. Therefore, in order to minimize the total durations for all nodes in the network to complete their temporal tasks, we need to find out the optimal strategy to minimize the charging delay for all nodes in the network. Specifically, in this paper we are aiming at finding the optimal stop locations, as well as the corresponding stop durations of the RFID reader to minimize the charging delay in the network. Fig. 3 shows an example of a reader stops at three locations to charge nodes in the network.

To formulate this charging problem, let us first assume the reader stops at several different locations and denote the location of its $j$-th stop as $\left(R_{x}^{j}, R_{y}^{j}\right)$ and the corresponding stop duration as $t_{j}$. Consequently, the distance between the $j$-th reader stop location and node $i$ is $d_{i j}=$ $\sqrt{\left(W_{x}^{i}-R_{x}^{j}\right)^{2}+\left(W_{y}^{i}-R_{y}^{j}\right)^{2}}$, and the corresponding charging power is $P_{i j}=\frac{\alpha}{\left(d_{i j}+\beta\right)^{2}}$ based on Eq. 2. The accumulated energy of node $i$ at the $j$-th reader stop location is $P_{i j} t_{j}$. 


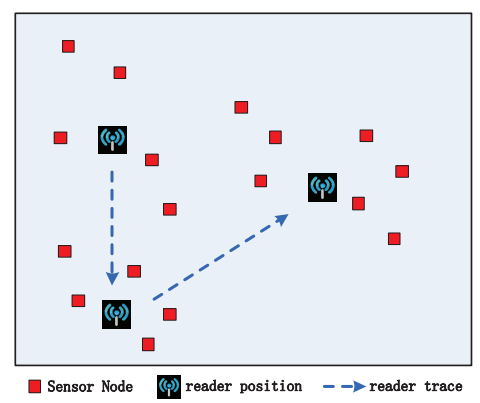

Fig. 3. Wireless rechargeable sensor network and reader stop positions

Given an area of interest with $N$ sensor nodes, the node charged energy threshold $\delta$, and the RFID reader can stop at any location in this continuous space, we can mathematically formulate the optimization problem of minimizing charge delay of all nodes in the network as:

$$
\begin{gathered}
\min \quad T=\sum_{j=1}^{\infty} t_{j} \\
\text { s.t. } \quad \sum_{j=1}^{\infty} P_{i j} t_{j} \geq \delta, \quad(i \in N) \\
P_{i j}=\frac{\alpha}{\left(d_{i j}+\beta\right)^{2}}, \quad(i \in N, j \in(1,2 \cdots \infty)) \\
d_{i j}=\sqrt{\left(W_{x}^{i}-R_{x}^{j}\right)^{2}+\left(W_{y}^{i}-R_{y}^{j}\right)^{2}} .
\end{gathered}
$$

To find the optimal reader stop locations and the corresponding stop durations for Eq. 3, the most straightforward method is to include all possible stop locations of the RFID reader in the Linear Programming (LP) solver. However, this simple method incurs very high computational overhead and is not suitable for most practical applications. Therefore in the following subsections, we introduce a set of new techniques to effectively reduce the search space of reader stop locations while still maintaining provable system performance.

\section{B. The Smallest Enclosing Space}

Inspired by Welzl's work on the smallest enclosing disk [21], we observe that the search space of reader stop locations can be first reduced to a Smallest Enclosing Space (SES), which is the smallest circular disk that covers all $N$ wireless rechargeable sensor nodes in the network. The computational complexity to find such a unique disk for a particular network deployment is $\mathcal{O}(N)$ [22]. Consequently, we have the following lemma on the search space of reader stop locations:

Lemma 1: To minimize the total charging delay for a network, the reader must stop within the Smallest Enclosing Space $C$ that covers all nodes in the network.

Proof: We prove this lemma by contradiction. Assuming there is an optimal stop location for the reader that is outside the SES area (denoted as $k$ in Fig. 4), then we could prove that there always exists a location within SES, which can lead to a smaller charging delay. Let the center of SES be $O_{C}$, and $h$

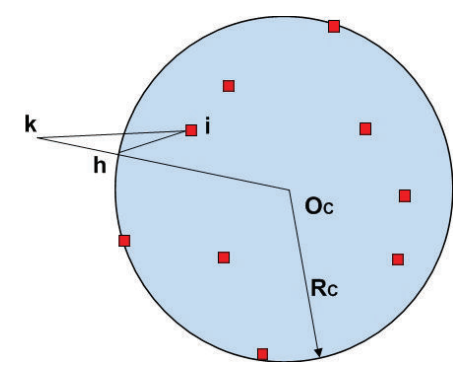

Fig. 4. The reader must stop within SES disk

be the intersecting point where line segment $\left[k, O_{C}\right]$ cuts the SES circle, for any node $i$ within SES, we have $d_{i h}<d_{i k}$ and consequently $P_{i h}>P_{i k}$. Therefore, by relocating the reader from location $k$ to $h$, we can always increase the charging power for any node $i$ and reduce the charging delay at node $i$. Consequently, we prove $k$ is not possible to be an optimal stop location for the reader and this concludes the proof.

\section{Discretizing Charging Power within SES}

Based on Lemma 1, we reduce the search space of reader stop locations from a two-dimensional space to a smaller enclosing circular area $C$. However, the potential reader stop locations within the SES $C$ is still infinite and we still cannot effectively apply the LP method in Section III-A to find out the optimal reader stop locations and the corresponding stop durations. In this subsection, we introduce a novel method that effectively discretizes the charging power within SES, and consequently reduce the computational complexity significantly by only considering a limited number of potential reader stop locations to the LP formulation in Section III-A.

As the search space for potential stop locations is reduced to SES, the distance between a node $i$ in the network and the potential stop locations of the reader is also reduced to a limited range. Let $O_{C}$ and $R_{C}$ be the center and the radius of the SES disk $C$, and $D_{i, O_{C}}$ be the distance from node $i$ to $O_{C}$, we can express the minimal and maximal distances from node $i$ to all possible reader stop locations as:

$$
\begin{aligned}
D_{i}^{\min } & =0, \\
D_{i}^{\max } & =D_{i, O_{C}}+R_{C} .
\end{aligned}
$$

With this range of charging distances and the charging model in Eq. 5, we can obtain the corresponding charging power range as:

$$
\begin{aligned}
P_{i}^{\min } & =\alpha /\left(D_{i, O_{C}}+R_{C}+\beta\right)^{2}, \\
P_{i}^{\max } & =\alpha / \beta^{2} .
\end{aligned}
$$

To discretize charging power within SES at a node $i$, we divide SES $C$ with respect to node $i$ by drawing $G_{i}$ number of concentric circles centered at node $i$ with increasing radius of $D_{i}[1], D_{i}[2], \ldots, D_{i}\left[G_{i}\right]$, such that the difference of charging powers between neighboring circles is less than a threshold value $\varepsilon(0<\varepsilon<1)$. Consequently, for a region between a pair of neighboring circles, the difference of charging powers to node $i$ is less than the threshold $\varepsilon$, and can be represented by a discretized charging power sequence $P_{i}[1], P_{i}[2], \ldots$, 


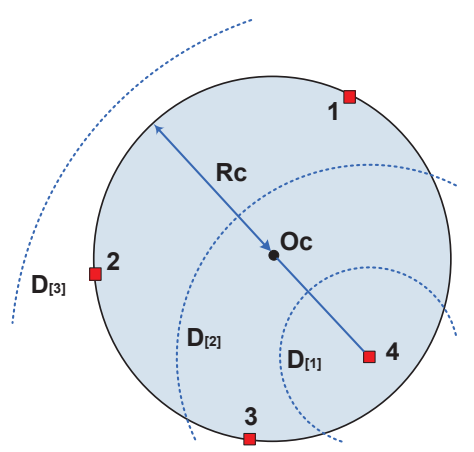

Fig. 5. A sequence of concentric circles with discretized charging powers with origin of node 4

$P_{i}\left[G_{i}\right]$, where $P_{i}[g]$ is:

$$
P_{i}[g]=P_{i}^{\max }(1+\varepsilon)^{-g} \quad\left(1 \leq g \leq G_{i}\right),
$$

Fig. 5 visualizes the charging power discretization process. For example of node 4, combined with Eq. 11, the charging power on the circle of $D[1]$ is $P_{4}[1]=P_{4}^{\max }(1+\varepsilon)^{-1}$, as $D[2]$ with $P_{4}[2]=P_{4}^{\max }(1+\varepsilon)^{-2}$. Hence, for the region between $D[1]$ and $D[2]$, the charging power is bounded by $P_{4}^{\max }(1+$ $\varepsilon)^{-1}$ and $P_{4}^{\max }(1+\varepsilon)^{-2}$, with a difference of charging power to node 4 is less than the threshold $\varepsilon$.

The total number of concentric circles $G_{i}$ for node $i$ therefore is decided by the smallest circle, which is centered at node $i$ and covers the whole SES $C$. In other words, $G_{i}$ is the smallest integer number satisfies the following condition: $P_{i}\left[G_{i}\right]=P_{i}^{\max }(1+\varepsilon)^{-G_{i}} \leq P_{i}^{\min }$. Consequently, combined with Eq. 9 and Eq. 10, we can determine $G_{i}$ with the following equation:

$$
G_{i}=\left\lceil\frac{\ln \left(\frac{P_{i}^{\max }}{P_{i}^{\min }}\right)}{\ln (1+\varepsilon)}\right\rceil=\left\lceil\frac{2 \ln \left(1+\frac{1}{\beta}\left(D_{i, O_{C}}+R_{C}\right)\right)}{\ln (1+\varepsilon)}\right\rceil
$$

As Fig. 5 shows, $D_{4}[3]$ is the smallest circle centered at node 4 and covers the whole SES disk, and we have $G_{4}=3$.

Similarly for all nodes in the network, we can apply the same charging power discretization process on the SES and obtain a finite number of regions that are bounded by concentric circles, which circles are originated from all nodes in the network. The total number of regions, denoted as $S$, can be determined as follows. Each region's boundaries are determined by the circle of SES disk $C$ and the concentric circles centered at all nodes. The largest number of the concentric circles within SES for each node $i$ is $G_{i}-1$ and there is only one SES disk circle, so the total number of arcs within SES is $Z=1+\sum_{i \in N}\left(G_{i}-1\right)$. By [23], the largest number of $S$, which is determined by $Z$ arcs has an upper bound:

$$
S \leq Z^{2}-Z+2 .
$$

As an example, Fig. 6 shows the concentric circles that are originated from four nodes in the network and divide the whole SES into 28 different regions.

For each region, denoted as $C_{s}$ (an example is illustrated in Fig. 6), the charging power difference from any location within this region to a neighboring region for all nodes in the

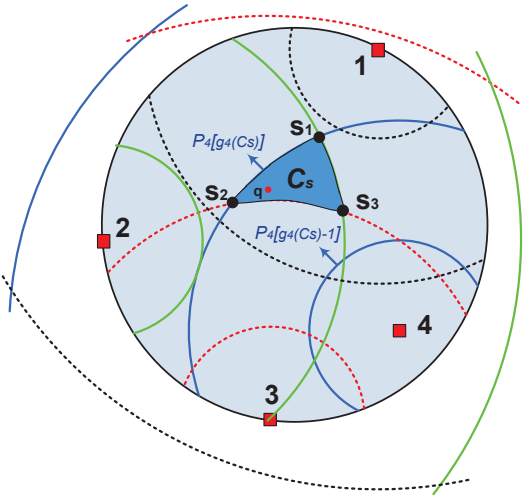

Fig. 6. An example of discretized charging regions within SES disk $C$

network is bounded by the threshold value $\varepsilon$. For any region $C_{s}$, it must be within the arc with a radius of $D_{i}[g]$ that is originated from a node $i$, and we denote the sequence number of this arc (w.r.t. to node $i$ ) as $g_{i}\left(C_{s}\right)$. Then, for any location $q \in C_{s}$, we have its charging power to node $i$ as:

$$
P_{i}\left[g_{i}\left(C_{s}\right)\right] \leq P_{i}[q] \leq P_{i}\left[g_{i}\left(C_{s}\right)-1\right] .
$$

Fig. 6 shows such example, any location $q$ in the region $C_{s}$, its charging power to node 4 is $P_{4}[q]$, and we have $P_{4}\left[g_{4}\left(C_{s}\right)\right] \leq P_{4}[q] \leq P_{4}\left[g_{4}\left(C_{s}\right)-1\right]$ (in this example $\left.g_{4}\left(C_{s}\right)=2\right)$. Since $\frac{P_{i}\left[g_{i}\left(C_{s}\right)-1\right]}{P_{i}\left[g_{i}\left(C_{s}\right)\right]}=1+\varepsilon$ by Eq.11, $P_{i}[q]$ has very tight lower and upper bounds of charging power with respect to node $i$.

Furthermore, for all $N$ nodes in the network, within a region $C_{s}$, the charging powers from any location in $C_{s}$ to all nodes are also bounded and can be represented as a $N$-tuple Charging Power Approximation vector $E_{s}=$ $\left[P_{1}\left(C_{s}\right), P_{2}\left(C_{s}\right), \ldots, P_{\mathrm{N}}\left(C_{s}\right)\right]$, where the $i$-th element is:

$$
P_{i}\left(C_{s}\right)=P\left[g_{i}\left(C_{s}\right)\right] \text {. }
$$

Fig. 6 visualizes an example, the charging power approximation vector for the region $C_{s}$ (enclosed by arcs $s_{1}, s_{2}$ and $\left.s_{3}\right)$ is $\left[P_{1}\left(C_{s}\right), P_{2}\left(C_{s}\right), P_{3}\left(C_{s}\right), P_{4}\left(C_{s}\right)\right]=$ $[P[2], P[2], P[3], P[2]]$, where the $i$-th element in the vector is the lower charging power bound from any point in this region to node $i$. For any point $q \in C_{s}$ and its corresponding charging power approximation vector $E_{s}$, we have:

$$
P_{i}\left(C_{s}\right) \geq \frac{P_{i}(q)}{(1+\varepsilon)} .
$$

Consequently, all such regions within SES can serve as a finite number of potential reader stop locations and use the LP method to solve Eq. 3 in Section III-A. The optimal total stop duration obtained by LP method after discretization has a $1 /(1-\varepsilon)$ approximated ratio to the theoretically optimal charging delay. The detailed proof is provided in Appendix, and we further verify this approximated ratio through extensive evaluations in Section IV.

\section{Reader Stop Location Merging Design}

After charging power discretization in Section III-C, we reduce the search space for the LP formulation in Section III-A from infinite space to finite number of potential reader stop 
locations. However, the optimal charging delay obtained may consist of a large number of reader stop locations. For example, Fig 7(a) shows a random deployment with 200 nodes and the corresponding optimal reader stop locations (denoted by stars in the figure). From Fig 7(a), we can see the reader have to stop at a very large number of locations (159 in this specific example) in order to achieve the optimal charging delay. However, for real-world applications, it is not very practical to move the reader among a large number of stop locations. Therefore, in this subsection, we introduce a reader stop location merging design that effectively reduces the number of stop locations for the reader while maintaining the charging delay increase within a specified threshold.
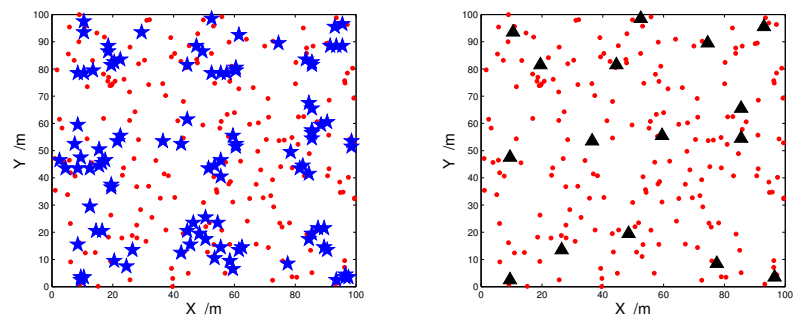

(a) Reader stop locations before (b) Reader stop locations after mergmerging (marked as stars) ing (marked as triangular dots)

Fig. 7. Illustration of reader stop locations before and after merging (sensor node marked as circular dots)

To merge the reader stop locations in the previous subsection, we use a well-known k-means clustering algorithm, called Lloyd's algorithm [24], to group reader stop locations into multiple clusters based on their geographical locations. The Lloyd's algorithm is a method of cluster analysis, which aims to partition $n$ observations into $k$ clusters in which each observation belongs to the cluster with the nearest mean.

For our reader stop location merging problem, the original $n$ observations in the Lloyd's algorithm is simply the total number of reader stop locations obtained using charging power approximation vectors in Section III-C. However, for the number of clusters to be formed ( $k$ in the Lloyd's algorithm), it is not obvious how to decide a suitable value. Therefore, in our reader stop location merging design, instead of asking system users to specify the $k$ value, we request system users to decide how much performance degradation they would tolerate so as to reduce the number of reader stop locations. Specifically, we let the system users to specify a threshold value $\theta$, such that the charging delay before stop location merging $\left(T_{\text {before }}\right)$ and after merging $\left(T_{\text {after }}\right)$ satisfy the following equation:

$$
\left|\frac{T_{\text {after }}-T_{\text {before }}}{T_{\text {before }}}\right| \leq \theta
$$

Essentially, $\theta$ decides the percentage of charging delay increase that the merging design can tolerate with respect to the delay before merging. Consequently, the charging delay after merging has an upper bound $(1+\theta) T_{\text {before }}$.

After introducing the delay threshold value $\theta$, the next question we need to answer is how to correlate $\theta$ with the number of clusters after merging (parameter $k$ in the Lloyd's

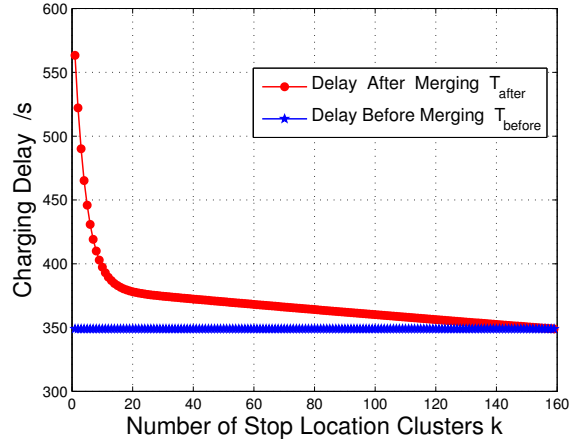

Fig. 8. Charging delay vs. the number of stop location clusters $k$

algorithm). To reveal the relationship between $\theta$ and charging delay after merging $\left(T_{\text {after }}\right)$, we use Fig. 8 to demonstrate how the charging delay changes with different value of merging parameter $k$ in the Lloyd's algorithm. From Fig. 8, we can see with the increasing number of stop location clusters from the Lloyd's algorithm, the charging delay decreases sharply when the number of clusters after merging is small and decreases slowly and approaches to the original charging delay when the number of clusters after merging is getting larger. Since the charging delay monotonically decreases with respect to the number of merged clusters, we can perform binary search to find the minimal $k$ value in the Lloyd's algorithm to satisfy the delay threshold $\theta$.

After obtaining the optimal $k$ value, we then need to select one representative stop location within each of the $k$ clusters. To select such a representative stop location within a cluster, we pick a location whose charging power vector is closest to the average charging power vector within this cluster. Specifically, assuming a cluster contains $M$ original reader stop locations, and their corresponding power vector is $E_{1}, E_{2}, \ldots, E_{M}$, respectively, we choose the representative location whose charging vector $E_{i}$ satisfies:

$$
E_{i}=\underset{E_{i} \in E}{\arg \min }\left(\left\|E_{i}-\left(\sum_{j=1}^{M} E_{j}\right) / M\right\|_{2}\right)
$$

Fig. 7(b) shows an example of reader stop locations after merging. If given a delay threshold $\theta=0.1$, we perform binary search to find the minimal $k$ value in the Lloyd's algorithm to satisfy this delay threshold is $k=16$. Then, we select one representative stop location within each of the $k$ clusters by Eq. 17 and mark these 16 representative locations as triangle dots in Fig. 7(b). Consequently, the charging delay after merging has an upper bound $(1+\theta) T_{\text {before }}=1.1 T_{\text {before }}$.

\section{E. Putting All Things Together}

To summarize our design, we divide it into following four main steps:

1) Finding the Smallest Enclosing Space (SES) that covers all nodes in the network,

2) Discretizing SES by charging powers for all nodes in the network, 
3) Solving Eq. 3 with discretized SES regions using LP methods, obtaining approximated optimal locations,

4) Merging these original approximated optimal locations into a smaller number of locations with a delay upper bound.

The approximation ratio for our design is $(1+\theta) /(1-\varepsilon)$ to the theoretically optimal charging delay.

The computation complexity of our design is determined by adding the following three parts. The first one (Section III-B) for finding the SES disk is $\mathcal{O}(N)$ [22]. The second one (Section III-C) for performing LP method to find approximated optimal locations is determined by the total number of partition regions, whose number has an upper bound as analyzed at the end of Section III-C. Hence, combined with Eq. 12 we obtain: $\mathcal{O}\left(Z^{2}\right)=\mathcal{O}\left(\left(\sum_{i \in N} G_{i}\right)^{2}\right)=\mathcal{O}\left((N / \varepsilon)^{2}\right)$. The third part (Section III-D) is determined by the number of iterations of performing LP method to find the minimal $k$ value using binary search $(\mathcal{O}(\log N)$ by [25]). As the complexity of the Lloyd's algorithm in each step of binary search is $\mathcal{O}(N)$ [24], the complexity of the third part is $\mathcal{O}(N \log N)$. So the total computation complexity of our design is $\mathcal{O}\left((N / \varepsilon)^{2}\right)$.

\section{Evaluation}

In this section, extensive simulations are conducted to evaluate our design under different network settings and reveal insights of system performance.

\section{A. Simulation Setup}

We assume wireless rechargeable sensor nodes are randomly deployed over a $100 m \times 100 m$ two-dimensional square area. The default number of sensor nodes is 100 . For the charging model (Eq. 2), we set $\alpha=36$ and $\beta=30$, which parameters are obtained by fitting of our experiment curve as mentioned in Section II-B. The charging power discretization threshold value is $\varepsilon=0.05$ and the stop location merging threshold is $\theta=0.05$. For each node, the energy threshold to function properly is $2 J$, which energy threshold is essential for WISP node to preform several sensing and computing tasks [9].

Each point in simulation figures is obtained by averaging 100 runs with different random seeds, node deployments and system parameters.

\section{B. Baseline Setup}

Since currently there is no existing works that are designed to minimize charging delay in wireless rechargeable sensor networks, to compare the system performance of our design, we introduce a baseline design that utilizes the concept of Set-Cover [26]. Essentially in this baseline design, the RFID reader tries to maximize the number of under-charged nodes in its surrounding region at individual stops.

In addition to the baseline design above, we also obtain the minimal charging delay by the fine-grained exhaustive search using the LP formulation in Eq. 3. This baseline is used to demonstrate the performance gap between our design and the theoretical charging delay lower bound.

\section{Performance Comparison}

In this subsection, we show the system performance under various designs with different system parameters including the charging power discretization threshold $\varepsilon$, stop location merging threshold $\theta$, the number of nodes and on-board capacitor size of node.

1) Impact of Charging Power Discretization Threshold $\varepsilon$ : As discussed in Section III-C, the charging power discretization threshold $\varepsilon$ affects the gap between the optimal charging delay and our approximated minimal delay with significantly reduced computational overhead. Therefore, we investigate the charging delay under different charging power discretization threshold values $\varepsilon$. As shown in Fig. 9, the charging delay remains constant for the optimal solution and baseline design as they are not affected by the charging power discretization threshold. In contrast, the charging delay of our approximated optimal design and stop location merging design increase with the increasing threshold value $\varepsilon$. This is because with a larger $\varepsilon$ value, the charging power vectors at individual discretized regions within SES become less bounded and consequently lead to longer charging delays with such larger charging power errors.

On the other hand, the charging power discretization threshold $\varepsilon$ also affects the computational overhead of our design as analyzed in Section III-E. To visualize such computational overhead, Fig. 10 shows the number of discretized charging regions under different charging power discretization threshold values. As shown in Fig. 10, the number of discretized charging regions decreases with the increasing charging power discretization threshold values, which in turn decreases the computational complexity as the number of potential reader stop locations decreases. For example, when $\varepsilon$ increases from 0.02 to 0.17 , the number of discretized charging regions decreases by $60.34 \%$. Therefore, based on the tolerable error range for the system, we can select the appropriate $\varepsilon$ value to reduce the computational overhead while satisfying the system requirements.

To compare with the baseline solutions, we can see the charging delays of our stop location merging design outperforms the set-cover-based baseline design at all charging power discretization threshold values. On average, our stop location merging design has $26.42 \%$ charging delay deduction when compared with the set-cover-based baseline design.

Furthermore, our design in Section III-D aims to ensure that the obtained charging delay has an approximation ratio of $\frac{(1+\theta)}{(1-\varepsilon)}$ with respect to the charging delay of optimal solution. In Fig. 9, we also show the charging delays of our design validate this approximation ratio. For example, when $\varepsilon=0.1$, the optimal delay is $303.4 s$ and the delay upper bound value is $\frac{(1+0.05)}{(1-0.1)} \times 303.4 s=354.0 s(\theta=0.05)$. At the same threshold value, the actual charging delay of our stop location merging design is $334.8 s$, therefore satisfies the delay bound.

2) Impact of Stop Location Merging Threshold $\theta$ : In this subsection, we study the impact of reader stop location merging threshold $\theta$ on the charging delays. In Section III-D, we 


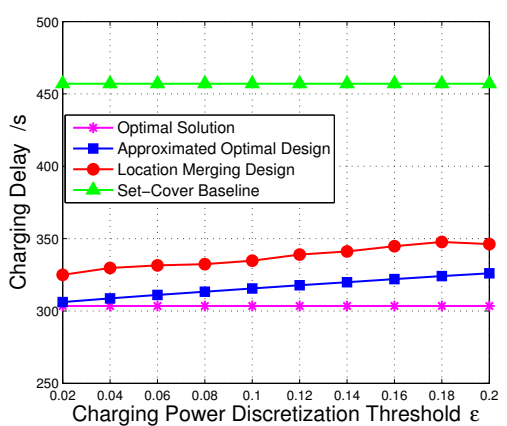

Fig. 9. Delay vs. Charging Power Discretization Threshold $\varepsilon$

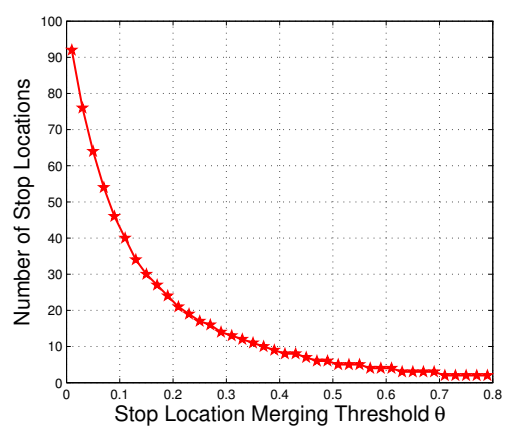

Fig. 12. Number of Reader Stop Locations vs. Location Merging Threshold $\theta$

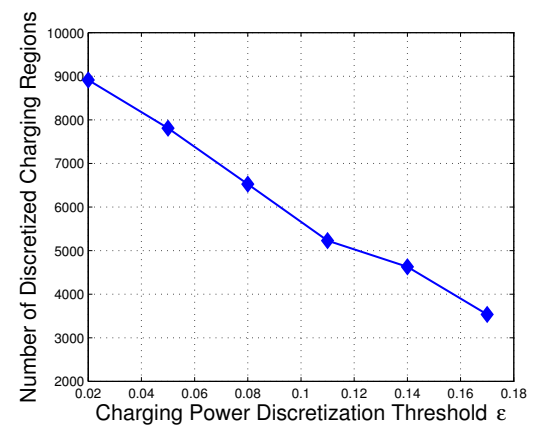

Fig. 10. Number of Discretized Regions vs Charging Power Discretization Threshold $\varepsilon$

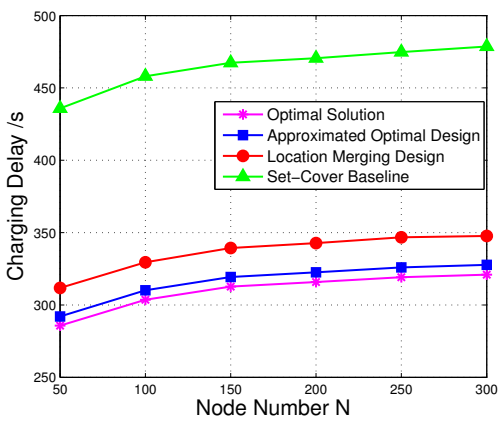

Fig. 13. Delay vs. Node Number

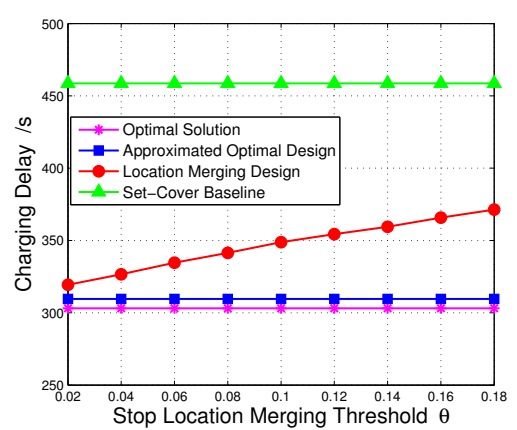

Fig. 11. Delay vs. Stop Location Merging Threshold $\theta$

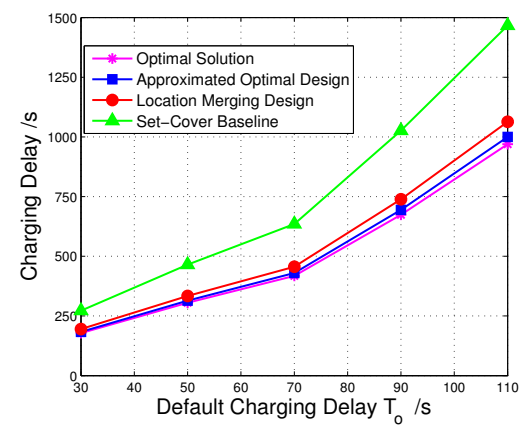

Fig. 14. Delay vs. Default Charging Delay introduce the stop location merging scheme to reduce the number of reader stop locations for practical systems and $\theta$ is used to control the charging delay bound. Fig. 11 shows the charging delays of different designs under varying $\theta$ values. From Fig. 11 we can see charging delays for the stop location merging design increase almost linearly with the increasing $\theta$ value, while all other designs are not affected and remain constant. However, even when $\theta=0.18$, the charging delay of our stop location merging design still outperforms the setcover-based design by $19.03 \%$. On average, our stop location merging design has $24.7 \%$ charging delay deduction when compared with the set-cover-based design.

To demonstrate the effectiveness of our stop location merging design, we also show the change of the number of reader stop locations under different $\theta$ values in Fig. 12. With an increasing value of $\theta$, the number of stop locations decreases sharply. Combined with the analysis of Fig. 11 and Fig. 12, our stop location merging design can significantly reduce the number of stop locations with a slight delay increase.

3) Impact of Node Number: We study the scalability of our design and investigate the charging delays with varying number of nodes in the network. Fig. 13 shows the impact of node density on the system performance. As the node density increases, the charging delays of all solutions also increase because there are more nodes needing to be charged. However, under all node density settings, it can be found that our designs significantly outperform the set-cover-based baseline design and closely approach the optimal charging delay. For example, when the number of nodes is 200 in the network, the charging delays for the optimal solution, approximated optimal design, stop location merging design and set-cover-based design are $312.7 s, 319.3 s, 339.4 s$ and $467.4 s$, respectively. Based on the delay of the optimal solution, we calculate the delay upper bound with approximation ratio as $\frac{(1+\theta)}{(1-\varepsilon)} \times 312.7 s=345.6 s$, and we can see the delay of our stop location merging design still satisfies this bound.

4) Impact of Varying Charging Models: We are interested in investigating the impact of different charging model parameters on the system performance. Particularly, we study the impact of varying on-board capacitor sizes on the network charging delays, which can provide guidance for the system designers when choosing appropriate capacitor size for their nodes. To simplify the description, we use the default charging delay $T_{0}$, which is defined as the charging time for the node when the charging power is maximal (right next to the RFID reader), to represent different on-board capacitor sizes. Fig. 14 shows the charging delay under different default charging delays $T_{0}$, which corresponding to different on-board capacitor sizes. From Fig. 14, we can see our designs outperform the setcover-based baseline design under all default charging delays. As $T_{0}$ becomes larger, the performance difference between our designs and set-cover design also becomes larger. For example, when the default charging delay is $T_{0}=30 \mathrm{~s}$, the difference between stop location merging design and set-cover baseline is $76.9 \mathrm{~s}$, while when the default charging delay is $T_{0}=110 \mathrm{~s}$, the corresponding difference is $404.1 s$. This increasing gap 

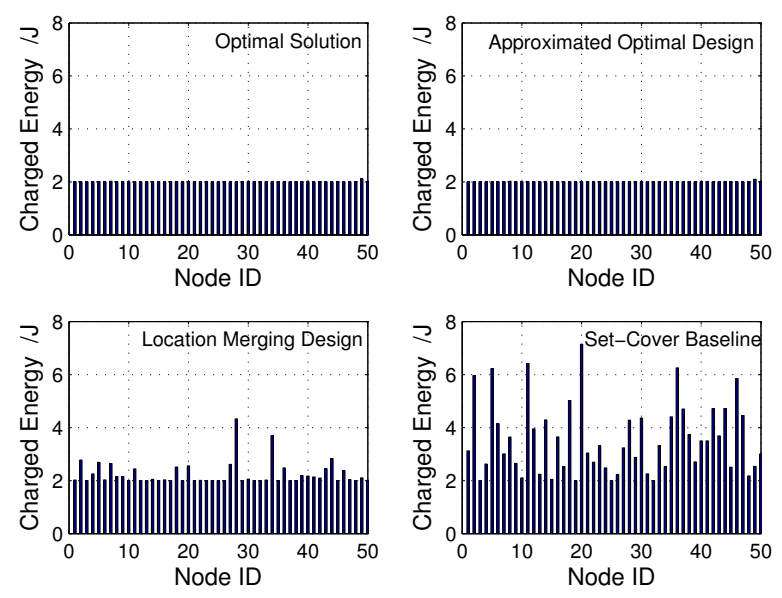

Fig. 15. Energy levels after charging with four solutions

of charging delays is because with a larger default charging delay $T_{0}$, each individual node needs more time to charge on average. As our stop location merging design provides a higher charging efficiency so that the total charging delay can be reduced more significantly.

\section{System Insights}

In order to reveal the insight about why our proposed design can significantly reduce charging delay, we conduct a simulation to observe the energy level on each node after charging with four solutions. Intuitively, the charging efficiency is high if each individual node is charged just with exact energy threshold $2 \mathrm{~J}$. The energy level for each node after charging is shown in Fig. 15. We can see in top two sub-figures of Fig. 15, most nodes exactly have levels of energy threshold, so the charging efficiency is high and a short charging delay is obtained. Comparing the bottom two sub-figures, the energy level of Set-Cover Baseline has a more various distribution, even many nodes are charged above $4 J$. Hence, a lot of energy is wasted by this charging solution and incurs a low charging efficiency. On the contrary, our stop location merging design charges energy to the nodes in a more balanced manner, so the charging efficiency is improved and our design can reduce charging delay significantly.

\section{RELATED WORK}

Recently, much research has focused on improving charging efficiency in wireless rechargeable sensor networks. In general, these works can be classified into two categories: hardware design and network charging coordination.

Many pioneer works have focused on hardware design to improve charging efficiency [6]-[8]. Kurs et al. [27] experimentally show that the overall output efficiency of charging multiple devices is larger than the output efficiency of charging each device individually by designing an enhanced technology. Sample et al. [9] design a scheme of analog circuitry for WISP node to obtain an efficient conversion of the incoming RF energy. Different from hardware design, our paper is focused on intra-network optimization, and the increase of charging efficiency originated from hardware domain could simultaneously improve the performance of our design.

From the view of networks, some recent works have investigated the network charging coordination to improve charging performance. He et al. [16] consider the static reader deployment in a wireless rechargeable sensor networks so that the nodes can harvest enough energy for continuous operation. In [17], Xie et al. consider the joint design of traveling path of mobile wireless charging vehicle (WCV), flow routing among the network, and charging time of WCV at each stopping point, and propose a near-optimal solution with guaranteed accuracy. The authors in [28] build a proofof-concept prototype of wireless charging system for sensor networks and conduct experiments to evaluate its feasibility and performance in small-scale networks. Bin et al. [29] investigate how to minimize charging cost by reducing energy consumption rate and improving recharging efficiency. All these works do not consider the charging delay on individual nodes, which is not negligible in practical applications and plays an important role in the overall system performance. So we consider the design to optimize the charging delay for the whole network, whose key objective is fundamentally different from previous works.

\section{CONCLUSiON}

We studied a general scenario of randomly deployed sensor nodes, where a reader moving as a point way in the network and staying for some duration at each location respectively to charge energy to the nodes. We used a novel solution to find the optimal set of stopping locations to achieve an approximated minimum of total charging time cost, and with a low search complexity. We first narrowed the search space into a smallest enclosed disk, and further divided this disk into finite number of regions with an accuracy of $\varepsilon$. Then we presented each region with a charging power vector and used LP method on these regions to find the optimal set of regions to position the reader with corresponding staying time. For practical consideration, we further proposed a location merging design to merge original stop locations into a smaller number with a delay upper bound. The total delay cost obtained by our design was $(1+\theta) /(1-\varepsilon)$ to the theoretically optimal one. We showed detailed illustration for the designs in simulation and achieved an approximated result with low search complexity. We also made some suggestions for reader stopping locations to minimize total delay cost. This solution could be used in other energy harvesting scenarios, with different types of sensor platforms or energy sources.

\section{ACKNOWLEDGEMENT}

This research was supported in part by NSFC under Grants 61222305, 61228302 and 61190110, NSFZJ under Grant R1100324, SRFDP under Grant 20120101110139, 111 Program under Grant B07031, 863 High-Tech Project under Grant 2011AA040101-1, SUTD-ZJU Collaboration Grant SUTDZJU/RES/03/2011. 


\section{REFERENCES}

[1] S. He, J. Chen, F. Jiang, D. Yau, G. Xing, and Y. Sun, "Energy provisioning in wireless rechargeable sensor networks," IEEE Transactions on Mobile Computing, to appear, 2013.

[2] L. He, Z. Yang, J. Pan, L. Cai, and J. Xu, "Evaluating service disciplines for mobile elements in wireless ad hoc sensor networks," in IEEE INFOCOM'12, 2012.

[3] Y. Shi and Y. Hou, "Optimal base station placement in wireless sensor networks," ACM Transactions on Sensor Networks, vol. 5, no. 4, p. 32, 2009.

[4] F. Jiang, S. He, P. Cheng, and J. Chen, "On optimal scheduling in wireless rechargeable sensor networks for stochastic event capture," in IEEE MASS'11, 2011.

[5] A. Kurs, A. Karalis, R. Moffatt, J. Joannopoulos, P. Fisher, and M. Soljačić, "Wireless power transfer via strongly coupled magnetic resonances," Science, vol. 317, no. 5834, pp. 83-86, 2007.

[6] "Powerharvester receivers," http://www.powercastco.com.

[7] M. Marroncelli, D. Trinchero, V. Lakafosis, and M. Tentzeris, "Concealable, low-cost paper-printed antennas for wisp-based rfids," in IEEE RFID'11, 2011.

[8] A. RamRakhyani, S. Mirabbasi, and M. Chiao, "Design and optimization of resonance-based efficient wireless power delivery systems for biomedical implants," IEEE Transactions on Biomedical Circuits and Systems, no. 99, pp. 1-1, 2011.

[9] A. Sample, D. Yeager, P. Powledge, A. Mamishev, and J. Smith, "Design of an rfid-based battery-free programmable sensing platform," IEEE Transactions on Instrumentation and Measurement, vol. 57, no. 11, pp. 2608-2615, 2008.

[10] "Diakinisis automates major distribution center with rfid," http://www. alientechnology.com/docs/CS_Diakinisis.pdf.

[11] T. Chen, T. Chen, and P. Wu, "On data collection using mobile robot in wireless sensor networks," IEEE Transactions on Systems, Man and Cybernetics, no. 99, pp. 1-12, 2011.

[12] M. Lin, H. Rowaihy, T. Bolbrock, G. Cao, and T. La Porta, "Data collection using rfid and a mobile reader,' in IEEE GLOBECOM'O8, 2008.

[13] O. Tekdas, V. Isler, J. Lim, and A. Terzis, "Using mobile robots to harvest data from sensor fields," IEEE Wireless Communications, vol. 16, no. 1, pp. 22-28, 2009.

[14] "Motorola mobile rfid reader: Case studies," http://www.motorola.com/ web/Business/Products/RFID/_Documents/Case_Studies/_StaticFiles/ ContinentalAG_Case_Study.pdf.

[15] M. Buettner, B. Greenstein, A. Sample, J. Smith, and D. Wetherall, "Demo: Rfid sensor networks with the intel wisp," in ACM Sensys'08, 2008.

[16] S. He, J. Chen, F. Jiang, D. Yau, G. Xing, and Y. Sun, "Energy provisioning in wireless rechargeable sensor networks," in IEEE INFOCOM'11, 2011.

[17] L. Xie, Y. Shi, Y. Hou, W. Lou, H. Sherali, and S. Midkiff, "On renewable sensor networks with wireless energy transfer: The multinode case," in IEEE SECON'12, 2012.

[18] N. Patwari, A. Hero III, M. Perkins, N. Correal, and R. O'dea, "Relative location estimation in wireless sensor networks," IEEE Transactions on Signal Processing, vol. 51, no. 8, pp. 2137-2148, 2003.

[19] N. Malhotra, M. Krasniewski, C. Yang, S. Bagchi, and W. Chappell, "Location estimation in ad hoc networks with directional antennas," in IEEE ICDCS'05, 2005.

[20] K. Ssu, C. Ou, and H. Jiau, "Localization with mobile anchor points in wireless sensor networks," IEEE Transactions on Vehicular Technology, vol. 54, no. 3, pp. 1187-1197, 2005.

[21] E. Welzl, "Smallest enclosing disks (balls and ellipsoids)," New Nesults and New Trends in Computer Science, 1991.

[22] N. Megiddo, "Linear-time algorithms for linear programming in r3 and related problems," in 23rd Annual Symposium on Foundations of Computer Science, 1983.

[23] M. De Berg, O. Cheong, and M. Van Kreveld, Computational Geometry: Algorithms and Applications. Springer-Verlag New York Inc., 2008.

[24] S. Lloyd, "Least squares quantization in pcm," IEEE Transactions on Information Theory, vol. 28, no. 2, pp. 129-137, 1982.

[25] T. Cormen, Introduction to algorithms. The MIT Press, 2001.

[26] N. Alon, D. Moshkovitz, and S. Safra, "Algorithmic construction of sets for k-restrictions," ACM Transactions on Algorithms, vol. 2, no. 2, pp. 153-177, 2006.
[27] A. Kurs, R. Moffatt, and M. Soljacic, "Simultaneous mid-range power transfer to multiple devices," Applied Physics Letters, vol. 96, no. 4, pp. 044 102-044 102, 2010.

[28] Y. Peng, Z. Li, W. Zhang, and D. Qiao, "Prolonging sensor network lifetime through wireless charging,' in IEEE RTSS'10, 2010.

[29] B. Tong, Z. Li, G. Wang, and W. Zhang, "How wireless power charging technology affects sensor network deployment and routing," in IEEE ICDCS'10, 2010.

\section{APPENDIX}

\section{A. Approximation Ratio Proof}

In this section, we prove that the optimal total stop duration obtained by LP method after discretization has a $1 /(1-\varepsilon)$ approximated ratio to the theoretically optimal charging delay under the condition that the reader only stops once. For a more general case when the reader has multiple stops, we verify the approximated ratio through extensive evaluations in Section IV.

Denote $q_{\text {opt }}$ as the theoretically optimal reader stop position (unknown), and $T_{o p t}, E_{o p t}$ the corresponding minimum charging delay and charging power vector, all of which are unknown. And we denote $E^{*}$ as the optimal one among all Charging Power Approximated (CPA) vectors $E_{s}, s=$ $1,2, \ldots, S$ to obtain minimum charging delay, which delay is denoted as $T^{*}$, so $T^{*}=\min \left\{T_{s}: s=1,2, \ldots, S\right\}$.

Theorem 1: For any reader stopping position $q$ with its corresponding charging power vector $E$ and optimal minimum charging delay $T$, denote $C_{s}$ as the region covering $q$ for a given $\varepsilon>0$. Then we calculate $E_{s}$ for this $C_{s}$, and claim its corresponding achievable optimal minimum charging delay $T_{s}$ (via LP method) is: $T_{s} \leq T /(1-\varepsilon)$.

Proof: Instead of using the optimal charging power vector $E_{s}$ for $C_{s}$, we use charging power vector $E$ to represent Charging Power Approximated vector of this region, which is obviously suboptimal. We denote $\hat{T}_{s}$ as the minimum delay obtained for $C_{s}$ under $E$, so $T_{s} \leq \hat{T}_{s}$. Then we only need to prove $\hat{T}_{s} \leq T /(1-\varepsilon)$. To prove this, combined with Eq. 4 , we calculate the total energy charged on node $i$ at time $T /(1-\varepsilon)$, which is:

$$
P_{i} \cdot T /(1-\varepsilon)>P_{i} \cdot T \geq \delta .
$$

The last inequality holds by the energy constraint in charging power vector for point $q$. Then the minimum delay $\hat{T}_{s}$ for subarea $C_{s}$ under $E$ is at most $T /(1-\varepsilon)$, and we have $T_{s} \leq$ $\hat{T}_{s} \leq T /(1-\varepsilon)$.

Theorem 2: With aforementioned $T^{*}$ and $T_{\text {opt }}$, we have $T^{*} \leq T_{\text {opt }} /(1-\varepsilon)$.

Proof: Consider a special case of Theorem 1 that a given reader stopping position is the theoretically optimal one $q_{o p t}$, with corresponding $E_{o p t}$ and $T_{o p t}$. Using the similar proof process, denote $C_{s}$ is the region covering $q_{o p t}$ with corresponding $E_{s}$, and we get $T_{s} \leq T_{o p t} /(1-\varepsilon)$. Hence for the optimal $E^{*}$ among all the Charging Power Approximated vectors, we have $T^{*} \leq T_{s} \leq T_{\text {opt }} /(1-\varepsilon)$. The proof is completed, and this theorem guarantees that the minimum optimal charging delay among all the $S$ Charging Power Approximated vectors is at most $T_{\text {opt }} /(1-\varepsilon)$. 